\title{
Electrochemical Oxygen Reduction Reaction on Ag Nanoparticles of Different Shapes
}

\author{
Tim Van Cleve, Emily Gibara, and Suljo Linic ${ }^{*[a]}$
}

Silver electrocatalysts are an attractive alternative to platinum for electrochemical oxygen reduction reaction in alkaline fuel cells. Recent advances in the synthesis of metal nanoparticles have enabled the design of silver nanoparticles of different shapes, terminated with different surface facets that exhibit different catalytic properties. In this contribution, we prepared spherical and cubic silver nanoparticle electrocatalysts and tested their electrocatalytic oxygen reduction reaction activity in $0.1 \mathrm{M}$ sodium hydroxide. Our work demonstrates that carbon-supported silver nanospheres and nanocubes of similar size exhibit similar ORR activity with the spheres slightly outperforming the cubes. In addition, we suggest possible reasons for the slightly enhanced activity of the nanospheres.

\section{Introduction}

Catalyzing the electrochemical oxygen reduction reaction (ORR) is critical for the design of highly efficient fuel cells. Although platinum has been the principal material of interest because of its high catalytic activity and superior stability- $\mathrm{Pt}$ and $\mathrm{Au}$ are the only stable metals under the conditions of electrocatalytic ORR in acidic electrolytes-the practical implementation of Pt-based electrodes has been hampered by their high cost. $^{[1]}$ While the superior electrocatalytic ORR activity of $\mathrm{Pt}$ compared to other metals persists in basic medium (with $\mathrm{OH}^{-}$ as the charge carrier), the number of materials that are stable under these conditions is significantly larger than in acid. ${ }^{[2]}$ For example, silver $(\mathrm{Ag})$ leaches in the form of oxidized $\mathrm{Ag}$ ions in acid under ORR conditions. On the other hand, Ag forms a stable oxide near ORR conditions in basic medium as shown in Figure 1. Ag is also currently approximately seventy times less expensive than $\mathrm{Pt} .{ }^{[3]}$ The main problem with $\mathrm{Ag}$ electrocatalysts is that in base these materials are at least an order of magnitude less active on a per-surface-site basis toward ORR than platinum. ${ }^{[4]}$

We recently demonstrated that $\mathrm{Ag}-\mathrm{Co}$ alloy nanoparticle electrocatalysts perform ORR in basic medium at a rate that is more than 5 times higher than the rate on pure Ag nanoparticles of identical size. ${ }^{[5]}$ We identified these materials based on an analysis of the ORR reaction mechanism on the $\mathrm{Ag}(111)$ surface at the elementary step level. ${ }^{[4,5]}$ This mechanistic analysis suggested that by perturbing the Ag surface atoms in such a way that they bind oxygenated surface intermediates slightly more strongly than the $\mathrm{Ag}(111)$ surface yields a more reactive Ag surface. Our studies suggested that the electronic communication between subsurface $\mathrm{Co}$ atoms and the surface $\mathrm{Ag}$

[a] T. Van Cleve, E. Gibara, Prof. S. Linic

Department of Chemical Engineering

University of Michigan

Ann Arbor, MI 48109-2136 (USA)

E-mail: linic@umich.edu

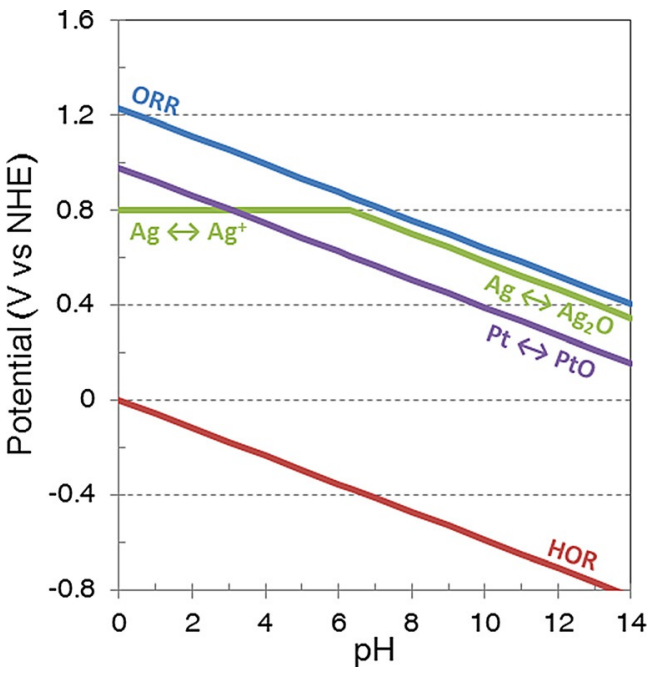

Figure 1. The Pourbaix diagram shows the equilibrium potentials for silver and platinum oxidization reactions as a function of the solution $\mathrm{pH}$ and potential with respect to the normal hydrogen electrode potential scale. At high potentials, oxidized species are favored making electrode stability a major concern in acidic electrolytes. Operating in alkaline conditions enables the use of more materials including silver as ORR catalysts.

atoms in the alloy nanoparticles electronically perturbs the $\mathrm{Ag}$ surface atoms yielding more reactive ORR materials. ${ }^{[5]}$

One way to accomplish the objective of creating Ag surface sites that bind oxygenated intermediates more strongly than the $\mathrm{Ag}(111)$ surface is, as mentioned above, to create suitable Ag alloys. ${ }^{[5,7]}$ An alternative approach is to control the distribution of surface facets exposed to the reactants and intermediates involved in ORR by controlling the morphology of $\mathrm{Ag}$ nanoparticles. ${ }^{[8]}$ For example, the $\mathrm{Ag}(100)$ surface binds oxygenated adsorbates more strongly that the $\mathrm{Ag}(111)$ surface. $^{[9]}$ As $\mathrm{Ag}$ nanocubes have a significantly larger concentration of the $A g(100)$ surface sites than the $A g$ nanospheres, we hypothesized that $\mathrm{Ag}$ nanocubes should be more reactive than 
nanospheres. In this contribution we have used colloidal synthetic routes to synthesize $\mathrm{Ag}$ nanoparticles of cubic and spherical shapes. We tested these nanostructures in electrochemical ORR in a basic environment. Contrary to our original hypothesis, we found that samples containing $\mathrm{Ag}$ spheres and Ag cubes exhibit similar performance with the $\mathrm{Ag}$ spheres slightly outperforming the cubes of equal size. We describe our findings below and suggest possible reasons for the superior activity of the nanospheres.

\section{Results and Discussion}

\section{Characterization of Ag nanoparticles}

The data in Figure 2 show the UV/Vis extinction spectra of various samples of $\mathrm{Ag}$ spheres and cubes prior to their deposition onto Vulcan XC 72 carbon. The extinction in the UV/Vis spectra is the consequence of the excitation of localized surface plas-

a

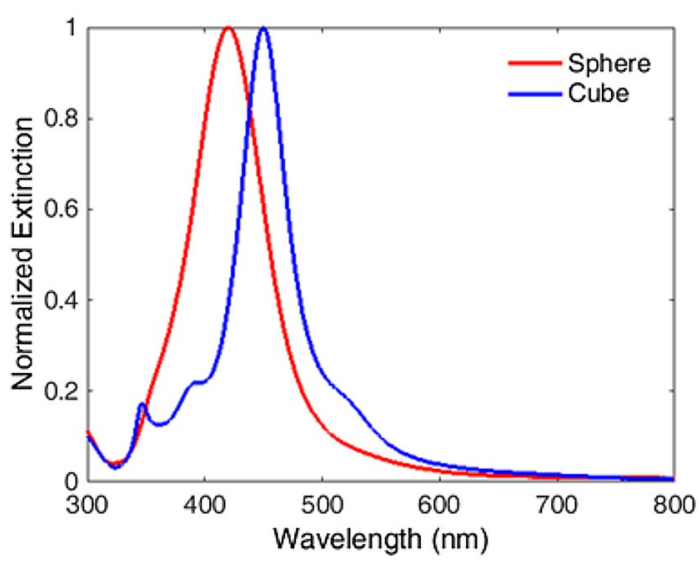

b

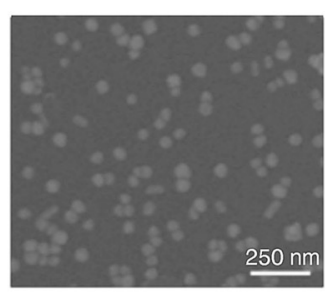

d

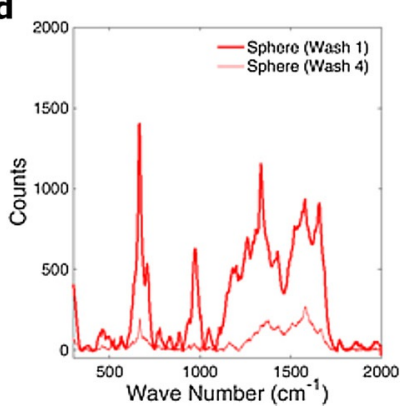

C

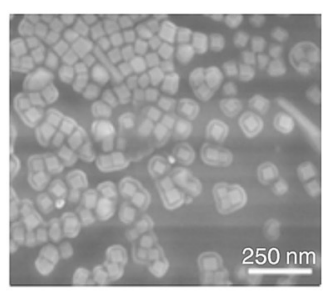

e

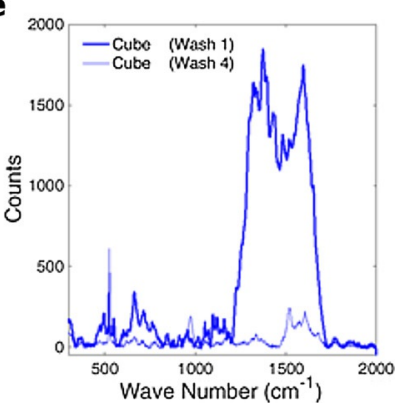

Figure 2. Extinction spectra (a) of representative Ag sphere and cube samples that were tested in our electrochemical experiments. SEM images of silver nanospheres (b) and nanocubes (c) particles deposited on Si substrate. SERS spectra Ag nanospheres (d) and Ag nanocubes (e) following multiple acetone rinses. mon resonance (LSPR) in Ag nanostructures. The wavelength and shape of the LSPR extinction peaks are characteristics of the shape and size of Ag nanoparticles. ${ }^{[10]}$ The appearance of the single peak in the Ag UV/Vis extinction spectra at around $400 \mathrm{~nm}$ is a characteristic of Ag spheres with $\approx 40 \mathrm{~nm}$ diameters, while the triple extinction peak is a distinguishing feature of nanoscopic Ag cubes. ${ }^{[10]}$ The UV/Vis extinction spectra are consistent with relatively pure samples of $\mathrm{Ag}$ cubes and spheres. Following the four acetone washes, the UV/Vis extinction spectra showed no changes compared to the original UV/ Vis spectra, suggesting minimal changes in particle morphology. The size and shape of $\mathrm{Ag}$ samples were also verified by SEM of nanoparticles deposited onto a silicon substrate, shown in Figure 2. Analysis of these micrographs revealed that Ag spheres had an average diameter of $43.7 \pm 8.5 \mathrm{~nm}$ and cubes had an average edge length of $40.8 \pm 5.4 \mathrm{~nm}$.

As we used colloidal synthesis methods that employ organic stabilizing agents (mainly polyvinylpyrrolidone, PVP), we attempted to remove these molecules before performing electrochemical tests. The removal of PVP was attempted by washing the sample in acetone multiple times as described above. Data in Figure $2 \mathrm{~d}$ and e show surface enhanced Raman spectra (SERS) of our Ag samples after the first and fourth washing step. The analysis of the SERS spectra suggests that there is a significant decrease in the PVP signal resulting from the washing process. We note that the Raman peaks in the range $1400-1600 \mathrm{~cm}^{-1}$ correspond to the $\mathrm{C}-\mathrm{C}$ stretches of PVP. ${ }^{[11]}$ The data also show that there is some residual PVP that was detected on Ag even after multiple washing steps; however, if comparing to the as-prepared samples and considering that these $\mathrm{Ag}$ nanostructures are excellent in enhancing Raman signal, the amount of residual PVP appears to be relatively small.

Data in Figure 3 show X-ray photoelectron spectroscopy (XPS) spectra of the Ag nanosphere and nanocube samples on carbon and the Ag-free carbon support. In this XPS analysis, among other elements, we focused on sulfur since it has been shown to affect the chemical activity of metals and it was used in the synthesis of the Ag cubes. ${ }^{[10,12]}$ The S2p XPS spectrum shows that sulfur is present in all the samples in the similar concentrations and chemical states suggesting that the main source of sulfur is the carbon support. Data in Figure 3 also show the Ag 3d XPS spectra. It is found that Ag is present in the metallic form.

\section{Electrochemical performance}

Linear sweep voltammetry experiments were performed on supported Ag nanoparticles (Ag/Vulcan XC72R) in the thin-film rotating-disk electrode ( $R D E)$ configuration to measure their oxygen reduction rate as a function of potential and rotation rate. The data in Figure 4 show the polarization behavior for representative $\mathrm{Ag}$ sphere and cube nanoparticle electrocatalysts. The polarization curves in Figure 4 were obtained by adjusting the kinetic current to an equal surface area basis (with a roughness factor of 1) as described in Ref. [8]. This was accomplished by first computing the raw kinetic current using 
a

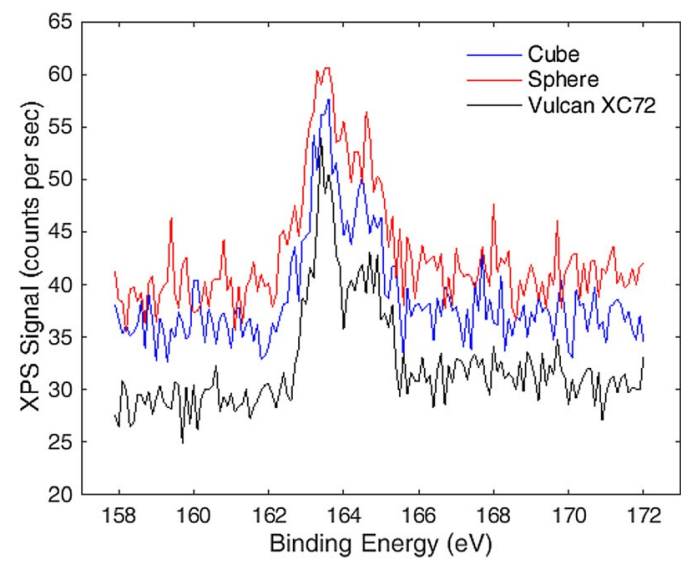

b

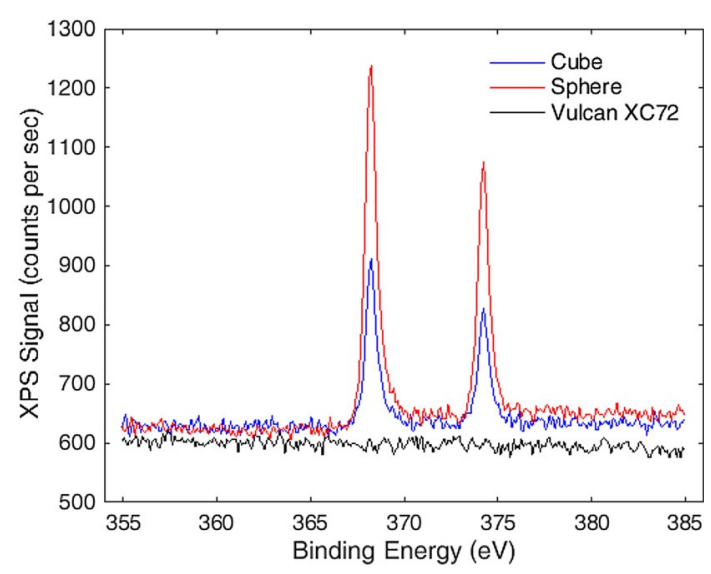

Figure 3. XPS spectra for S2p (a) and Ag 3d (b) core states measured for various Ag nanoparticle catalysts and carbon support.

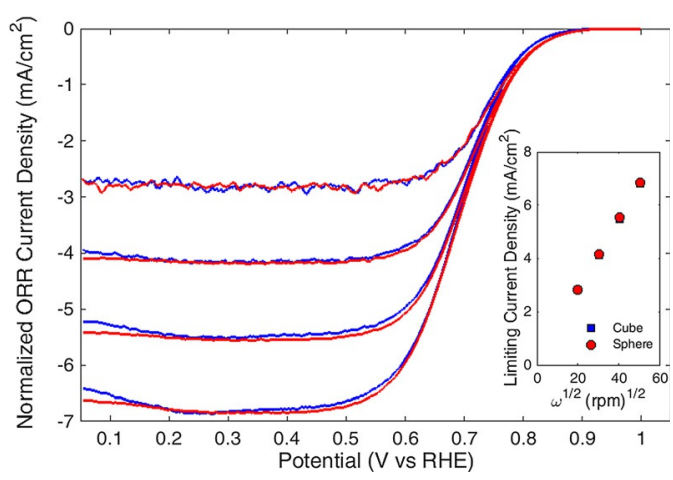

Figure 4. ORR current densities were measured for $\mathrm{Ag}$ nanospheres $(\longrightarrow)$ and nanocubes (-) as a function of potential ( $\mathrm{V}$ vs. RHE) at rotation rates of $400,900,1600$, and $2500 \mathrm{rpm}$. Both particle morphologies exhibited nearly equivalent mass-transfer limiting-current densities and similar selectivity towards complete $\mathrm{O}_{2}$ reduction to water. The slope of the Levich plot (inset) is consistent with a $4 \mathrm{e}^{-}$transfer mechanism.

Koutecky-Levich equation: $\left|i_{k}(V)\right|=\left(1 /|i(V)|-1 /\left|i_{L}\right|\right)^{-1}$, where $i$ is the raw measured current, $i_{k}$ is the raw kinetic current, and $i_{L}$ is the limiting current measured between 0.2 and $0.5 V_{R H E}$
$\left(\mathrm{V}_{\mathrm{RHE}}=\right.$ potential relative to the reversible hydrogen electrode (RHE). The raw kinetic current was then normalized to the electrochemical surface area (ESCA). We describe further below how ESCA was measured. The normalized kinetic current density $\left(j_{k}=\left|i_{k}\right| / E C S A\right)$ was then used to regenerate the RDE current density $(j)$ curves on a superficial area basis as: $|j|=\left(1 / j_{k}+\right.$ $\left.A_{\text {disk }} /\left|i_{L}\right|\right)^{-1}$.

At high potentials, ORR is limited by the intrinsic activity of the electrocatalysts and its loading, showing an exponential dependence of current on applied potential. At low potentials, the overall rate becomes limited by the transport of $\mathrm{O}_{2}$ to the surface which is a function of the RDE rotation rate. The kinetic rate of ORR can be determined by accounting for the effects of $\mathrm{O}_{2}$ transport to the electrode on the observed rate (current) as described in the Koutecky-Levich equation. The data in Levich plot (inset) shows the mass-transfer-limiting current density as a function of rotation rate. The slope of this line indicates that ORR proceeds through the $4 \mathrm{e}^{-}$pathway with very high selectivity to $\mathrm{H}_{2} \mathrm{O}$ for both electrocatalysts.

To compare the relative kinetic performance of different electrocatalysts, the rate of ORR was normalized by the number of electrocatalytic sites, or ECSA. This required a rigorous measurement of electrochemical surface area, i.e., the area at the reactive interface between the liquid electrolyte and metal electrode. The ECSA of Ag electrocatalysts was measured by utilizing the phenomenon of $\mathrm{Pb}$ underpotential deposition (UPD) onto Ag. Previous work has shown that by holding an Ag electrode slightly above the $\mathrm{Pb}^{2+} / \mathrm{Pb}$ reduction potential, a submonolayer ( $\approx 2 / 3$ monolayers) of $\mathrm{Pb}$ deposits onto the Ag surface. ${ }^{[13]}$ By scanning to higher potentials, the underpotentially deposited $\mathrm{Pb}$ submonolayer is dissolved, generating an oxidative current. By measuring the oxidative current, it is possible to directly measure the Ag ECSA by integrating the $\mathrm{Pb}_{\text {UPD }}$ oxidization peak. The data in Figure 5 show the oxidative currents generated during the oxidation of $\mathrm{Pb}$ sub monolayers on the surface of representative $\mathrm{Ag}$ cube and sphere electrocatalyst. To generate the plots of the oxidative current in Figure 6, the background resulting from capacitive charging of the carbon support was removed. We note that at the poten-

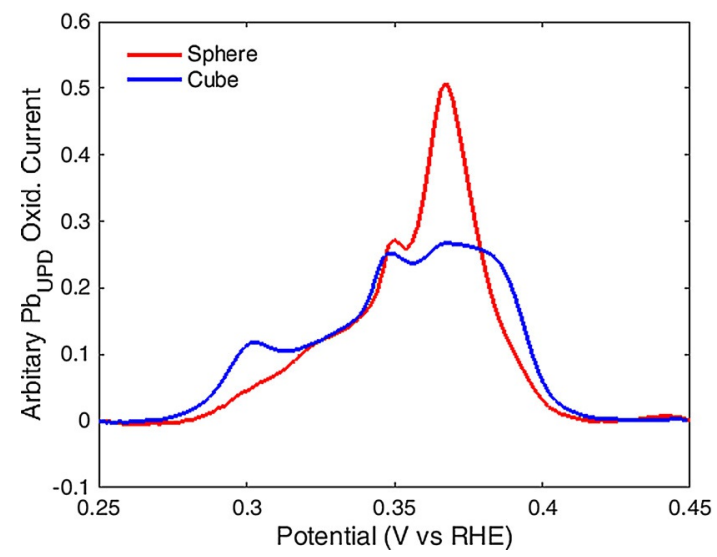

Figure 5. The background-corrected Pb UPD oxidization curves for silver nanocubes $(-)$ and nanospheres $(-)$ in $0.1 \mathrm{M} \mathrm{NaOH}$ show different features over the potential range of $0.25-0.45 \mathrm{~V}$. 
ORR Activity on Ag Nanoparticles at $0.8 \mathrm{~V}$

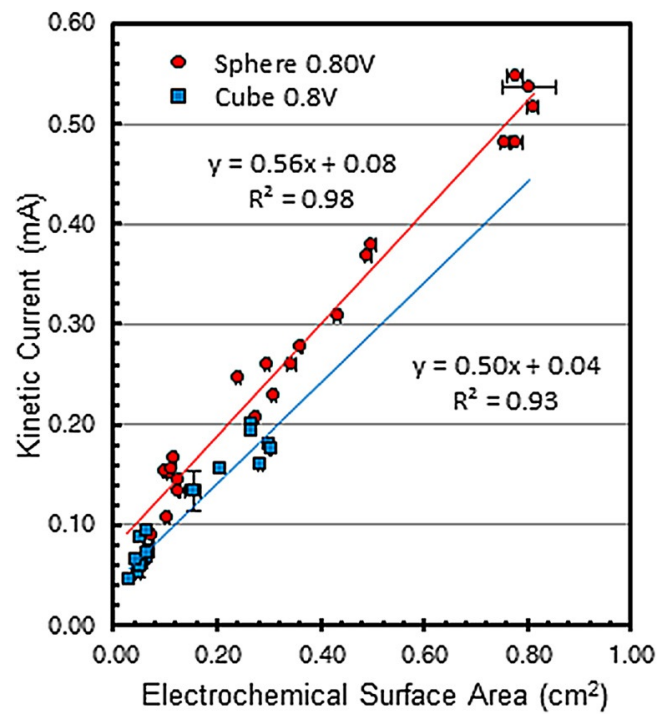

b

ORR Activity on Ag Nanoparticles at $0.85 \mathrm{~V}$

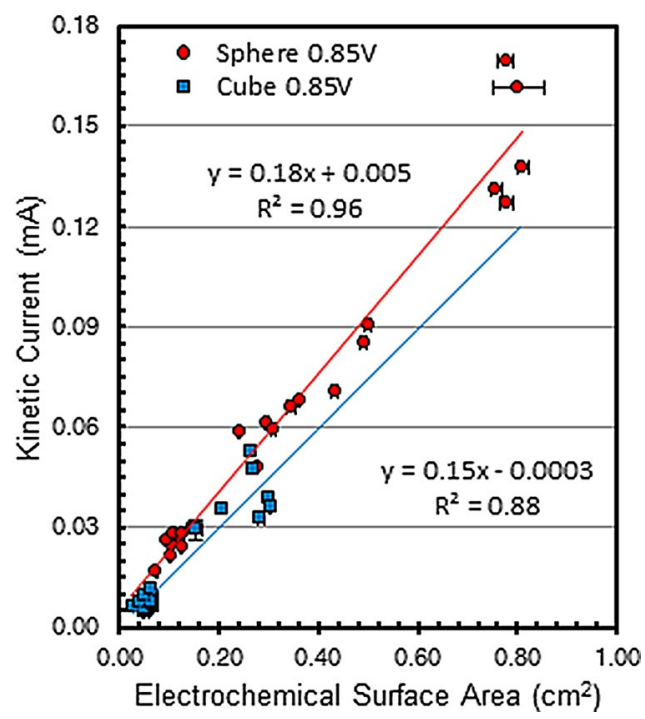

Figure 6. Alkaline ORR kinetic currents of supported silver nanosphere ( and nanocube ( $⿴$ ) electrocatalysts with different weight loadings at two different operating potentials: $0.80 \mathrm{~V}$ (a) and $0.85 \mathrm{~V}$ (b).

tial range where the oxidation and desorption of $\mathrm{Pb}$ from $\mathrm{Ag}$ surfaces takes place $(0.25-0.45 \mathrm{~V}$ vs. RHE) there were no other electrochemical processes and therefore no further background corrections were required. Multiple peaks in the oxidative current curves are associated with the $\mathrm{Pb}$ oxidation and desorption from the different surface facets of Ag. The multiple peaks allow us to quantify the contribution of different Ag surface facets to the electrochemical surface area. We will revisit to this issue further below in the discussion section.

The data in Figure 6 show the net kinetic currents (not normalized by the ESCA) for different samples of Ag nanoparticle electrocatalysts plotted against their electrochemical surface area at two different operating potentials $(0.80$ and $0.85 \mathrm{~V}$ vs. RHE). We note that unlike for Pt electrodes, where it is customary to report currents at $0.9 \mathrm{~V}$ vs. $\mathrm{RHE}, \mathrm{Ag}$-based electrodes exhibit lower ORR activity and the currents are usually reported at 0.85 and $0.80 \mathrm{~V}$. The kinetic current was obtained as described above using Koutecky-Levich equation. The data shows that oxygen reduction rate over this range of metal nanoparticle loadings scales linearly with the electrochemical surface area. This linear scaling suggests that the electrode design allows for a rigorous deconvolution and measurements of kinetic currents, which is critical if different electrocatalysts are to be compared using the RDE setup.

The slope of the lines in Figure 6 corresponds to the average specific activity (the ORR rate per electrochemical surface area) of the nanoparticles over this range of surface areas. By comparing these slopes at various potentials, it is possible to compare the performance of different Ag nanoparticle electrocatalysts. From the specific activities calculated for these silver nanoparticle samples, we observe that spheres are approximately $12 \pm 8 \%$ and $18 \pm 10 \%$ more active than cubes at 0.80 and $0.85 \mathrm{~V}$ on the reversible hydrogen electrode potential scale.

Data in Figure 6 show relatively small differences in ORR activity between Ag nanospheres and nanocubes. One plausible explanation is that nanoparticles undergo morphological changes in the electrochemical environment leading to similar surface structures and therefore similar activities. To address this possibility we performed in situ electrochemical adsorption/desorption measurements to investigate potential electrocatalyst restructuring during the course of the measurement. Whereas cyclic-voltammetry measurements on Ag nanoparticles in deaerated electrolyte did not yield any significant electrochemical adsorption features, both Ag structures exhibited distinct $\mathrm{Pb}_{\text {UPD }}$ stripping features, characteristic of the presence and persistence of different Ag surface facets.

The data in Figure 5 show the $\mathrm{Pb}_{\mathrm{UPD}}$ oxidative stripping spectra for the two Ag nanoparticle samples as a function of electrochemical potential measured after a set of electrochemical measurements were performed on the samples. The $\mathrm{Pb}$ stripping spectra show that the Ag surfaces in the two electrocatalysts are different from each other. Previous measurements on well-defined $\mathrm{Ag}(111)$ and $\mathrm{Ag}(100)$ single crystal surfaces allow us to assign the stripping features to a particular Ag surface facet. ${ }^{[13]}$ It has been demonstrated that the $\mathrm{Ag}(111)$ surface exhibits two sharp peaks located at +130 and $+155 \mathrm{mV}$ relative to $\mathrm{Pb} / \mathrm{Pb}^{2+}$ reduction potential (corresponding to 0.34 and $0.37 \mathrm{~V}_{\mathrm{RHE}}$ ), whereas the $\mathrm{Ag}(100)$ surface exhibits three peaks: a short broad feature located at $+50 \mathrm{mV}\left(0.26 \mathrm{~V}_{\mathrm{RHE}}\right)$, a sharp peak located at $+100 \mathrm{mV}\left(0.31 \mathrm{~V}_{\mathrm{RHE}}\right)$, and a broad peak located at approximately $+135 \mathrm{mV}\left(0.36 \mathrm{~V}_{\mathrm{RHE}}\right) .{ }^{[13]}$

Utilizing the $\mathrm{Pb}_{\text {UPD }}$ stripping spectra, it is possible to estimate the relative abundance of the $\mathrm{Ag}(111)$ and $\mathrm{Ag}(100)$ sites on Ag cubes and spheres. To accomplish this, we deconvoluted the measured $\mathrm{Pb}$ stripping spectra in terms of the linear combination of the previously reported and discussed $\mathrm{Ag}(111)$ and $\mathrm{Ag}(100) \mathrm{Pb}$ spectra. ${ }^{[13 a]}$ The concentration of various surface facets on our nanostructures was determined by minimizing 
the sum of square errors between the deconvoluted and experimental spectra. Not surprisingly, we found that Ag nanocube samples exhibit largely $(100)$ character $(\approx 80-95 \%)$ whereas Ag spheres contained significant fractions of both surfaces with $\mathrm{Ag}(100)$ surface facets covering approximately 40 $45 \%$ of the surface.

The observation that the $\mathrm{Ag}$ spheres are more active that Ag cubes is in contradiction with our proposed hypothesis that $\mathrm{Ag}$ surfaces that bind oxygenated intermediates slightly more strongly than the clean $\mathrm{Ag}(111)$ should exhibit higher ORR activity than $\mathrm{Ag}(111)$. We postulate that the reason for this contradiction is that the $\mathrm{Ag}(100)$ surface binds oxygenated intermediates, such as $\mathrm{OH}^{\circ}$, too strongly and that under relevant high potential ORR conditions these sites are poisoned by these intermediates. This is supported by DFT calculations which showed that $\mathrm{Ag}(100)$ binds $\mathrm{OH}$ stronger than $\mathrm{Ag}(111)$ by $\approx 0.5 \mathrm{eV}$, suggesting that $\mathrm{Ag}(100)$ is more likely to be poisoned at high potentials and therefore less catalytically active than the $A g(111)$ surface. ${ }^{[9 a, 14]}$ We also note that Blizanac and coworkers demonstrated using single crystal $\mathrm{Ag}(111)$ and $\mathrm{Ag}(100)$ electrodes that $\mathrm{Ag}(111)$ was $\approx 33$ and $50 \%$ more active than $\mathrm{Ag}(100)$ at 0.80 and $0.85 \mathrm{~V}$ vs. RHE, respectively, for ORR performed in $0.1 \mathrm{M} \mathrm{KOH}^{[4 a]}$ If we assume cubes are entirely terminated by $\mathrm{Ag}(100)$ sites and spheres have $45 \% \mathrm{Ag}(100)$ and $55 \% \mathrm{Ag}(111)$ surface coverage, using these previously reported ORR rate numbers we compute that the spheres are expected to be more active than cubes by $\approx 18-27 \%$ at potentials between 0.80 and $0.85 \mathrm{~V}_{\mathrm{RHE}}$, which is relatively consistent with our findings. ${ }^{[4 a]}$ This work suggests the intrinsic ORR activity of $\mathrm{Ag}$ nanostructures cannot be improved by replacing $\mathrm{Ag}(111)$ with $\mathrm{Ag}(100)$ surface sites.

\section{Conclusions}

We have demonstrated that carbon-supported $40 \mathrm{~nm} \mathrm{Ag}$ spheres are slightly more catalytically active towards alkaline oxygen reduction reaction (ORR) than carbon-supported $40 \mathrm{~nm}$ Ag cubes. Ex situ and electrochemical characterization have shown that Ag nanoparticles maintain their distinct morphologies throughout catalyst preparation and testing. We observed that the relative ORR activity enhancement of $\mathrm{Ag}$ spheres compared to $\mathrm{Ag}$ cubes is consistent with the abundance of the $\mathrm{Ag}(111)$ surface facets compared to $\mathrm{Ag}(100)$ on the spheres.

\section{Experimental Section}

\section{Electrocatalyst preparation}

Silver nanospheres were synthesized following a previously established procedure. ${ }^{[8,15]}$ In general, ethylene glycol (10 mL, JT Baker) was added to a vial and heated for $45-60 \mathrm{~min}$ at $160^{\circ} \mathrm{C}$ in a silicone oil bath. Then, $3 \mathrm{~mL}$ solutions of $600 \mathrm{~mm}$ PVP (Sigma-Aldrich; $M_{\mathrm{w}}=55000$ ) and $100 \mathrm{~mm} \mathrm{AgNO}_{3}$ (Sigma-Aldrich) in ethylene glycol were prepared and dropwise added to the heated vial. The vial was capped and after approximately $1 \mathrm{~h}$ removed from the oil bath and quenched with cool water.

Silver cubes were synthesized by a method developed by Xia. ${ }^{[10 b]}$ Ethylene glycol $(5 \mathrm{~mL})$ was heated to $140-150^{\circ} \mathrm{C}$. After $45 \mathrm{~min}$,
$30 \mathrm{~mm} \mathrm{Na} 2 \mathrm{~S}$ solution $(80 \mu \mathrm{L}$ ) was added to ethylene glycol. A few minutes later, $0.28 \mathrm{M} \mathrm{AgNO}_{3}$ solution $(0.5 \mathrm{~mL})$ and $0.18 \mathrm{M}$ PVP solution $(1.5 \mathrm{~mL})$ were quickly added to the vial. The vial was capped and the solution was allowed to react for approximately $45 \mathrm{~min}$ after which it was removed from the oil bath to inhibit the further nanoparticle growth.

The solutions containing Ag nanoparticles were washed four times with acetone to remove capping agents, solvent, and unreacted reagents. After each washing procedure, Ag nanoparticles were collected by centrifugation at $7000 \mathrm{rpm}$ for $20 \mathrm{~min}$. The effluent was discarded and $\mathrm{Ag}$ nanoparticle suspensions were reconstituted in a small volume $(2-5 \mathrm{~mL})$ of ethanol prior to the next acetone wash. After the final centrifugation, Ag nanoparticles were re-suspended in pure ethanol and deposited onto Vulcan XC72R powder (Cabot, pretreated for at least $2 \mathrm{~h}$ in $\mathrm{H}_{2}$ at $400^{\circ} \mathrm{C}$ ). The catalyst solutions were dried overnight in a heating oven.

\section{Characterization}

UV/Vis extinction spectroscopy experiments were performed using a Thermo Scientific Evolution 300 UV/Vis spectrophotometer with a Xenon lamp source to measure the extinction spectra of dilute $\mathrm{Ag}$ nanoparticle suspensions. UV/Vis spectra were collected between wavelengths of 300 and $800 \mathrm{~nm}$ at a rate of $600 \mathrm{~nm} \mathrm{~min}^{-1}$ and were normalized by the peak signal intensity to facilitate comparison of samples with different concentrations. SEM was performed on Ag nanoparticles deposited directly on a clean Si chip $\left(\approx 1 \mathrm{~cm}^{2}\right.$; rinsed with piranha solution and dried) using a FEI NOVA Nanolab operating in secondary electron detection mode. Micrographs were obtained by using a TLD (through-the-lens detector) and the accelerating voltage was $15 \mathrm{kV}$. SERS experiments were measured on $\mathrm{Ag}$ nanoparticle on $\mathrm{Si}$ substrate using a Horiba Jobin-Yvon LabRAM HR system. SERS spectra were acquired over $300-2000 \mathrm{~cm}^{-1}$ range using a $532 \mathrm{~nm}$ laser with $5 \mathrm{~s}$ acquisition time and by averaging the signal over 10 consecutive acquisitions. XPS experiments were performed on Ag nanoparticle catalysts on Vulcan XC72R support using a Kratos Axis Ultra XPS with monochromatic $\mathrm{Al}_{\mathrm{Ka}}(1486.69 \mathrm{eV}) \mathrm{X}$-ray source. As-prepared Ag/C samples were heated overnight in an UHV system to remove residual moisture before being directly transferred to a vacuum chamber (pressure of $\approx 10^{-9}$ torr) for analysis. XPS spectra were measured at every $0.1 \mathrm{eV}$ over the ranges of 158-172 eV, 277-300 eV, and 355$385 \mathrm{eV}$ to observe S2p, C 1s, and Ag3d peaks. XPS signals were normalized by their acquisition times $(10.6,6.5$, and $7.5 \mathrm{~s}$, for $\mathrm{S}, \mathrm{C}$, and $\mathrm{Ag}$ peaks, respectively). Charging effects were compensated using a flood gun. XPS binding energies were calibrated based on positioning the main $\mathrm{C} 1 \mathrm{~s}$ peak at $284.8 \mathrm{eV}$, which originates from the carbon support.

\section{Electrochemical measurements}

Electrochemical measurements were performed at RT in a custombuilt Teflon three-electrode cell with a Gamry Instruments Reference 3000 potentiostat/galvanostat/Frequency-Response-Analyzer. The reference $(\mathrm{Hg} / \mathrm{HgO}$ in $1 \mathrm{M} \mathrm{KOH}$, Radiometer Analytical) and counter electrodes (Pt wire, Alfa Aesar) were both in isolated compartments with long diffusion paths to the working electrode chamber. Working electrodes were prepared by sonicating the catalyst powders in $99.9 \%$ acetone (Fisher) at $1.50 \mathrm{mg} \mathrm{mL}^{-1}$ for more than an hour and dispersing $18 \mu \mathrm{L}$ onto a $5 \mathrm{~mm}$ glassy carbon electrode insert (Pine Instruments). These electrodes were placed 
into a Teflon RDE housing, which was attached to a rotator (Pine Instruments).

Electrolyte solutions of $0.1 \mathrm{M} \mathrm{NaOH}$ were prepared from ultrapure water $\left(18.2 \mathrm{M} \Omega \mathrm{cm}^{-1}\right.$ (Millipore)) and $99.99 \%$ sodium hydroxide (Alfa Aesar). The reaction rates were measured in the $\mathrm{O}_{2}$-saturated electrolyte using linear sweep voltammetry at rotation rates between $400-2500 \mathrm{rpm}$ at a scan rate of $10 \mathrm{mV} \mathrm{s}^{-1}$ to achieve steadystate rates. A consistent uncompensated resistance of $\approx 40 \Omega$ was measured via high-frequency impedance, and was corrected for in the polarization curves. All potentials are reported relative to the $\operatorname{RHE}\left(\mathrm{V}_{\mathrm{RHE}}=\mathrm{V}_{\mathrm{NHE}}+0.0591 \mathrm{pH}\right.$ (NHE, normal hydrogen electrode) $)$, calibrated by the $\mathrm{H}_{2}$ oxidation equilibrium. Electrochemical surface areas (ESCA) of $\mathrm{Ag}$ electrocatalysts were measured by $\mathrm{Pb}$ underpotential deposition immediately following the rate measurements. In these measurements $\mathrm{PbNO}_{3}$ was added to make $125 \mu \mathrm{M} \mathrm{Pb}{ }^{2+}$ in $0.1 \mathrm{M} \mathrm{NaOH}$ and the cell was deaerated by bubbling Ar for 15$20 \mathrm{~min}$. The electrode was repeatedly held at $0.20 \mathrm{~V}_{\mathrm{RHE}}$ and swept to $0.6 \mathrm{~V}_{\mathrm{RHE}}$ at $20 \mathrm{mV} \mathrm{s}^{-1}$. The stable voltammograms were integrated assuming $280 \mu \mathrm{Ccm}^{-2} \mathrm{Ag}^{[13]}$

\section{Acknowledgements}

We acknowledge support from the US DOE Office of Basic Energy Sciences, Division of Chemical Sciences (FG-02-05ER15686) and the University of Michigan Summer Undergraduate Research Program. We also acknowledge the University of Michigan Electron Microbeam Analysis Laboratory for use of the microscopy facilities. Finally, we acknowledge A. Holewinski for helpful discussions and experimental assistance.

Keywords: electrochemistry - nanostructures • oxygen • reduction $\cdot$ silver

[1] a) H. A. Gasteiger, S. S. Kocha, B. J. Sompalli, Appl. Catal. B 2005, 56, 9; b) I. E. L. Stephens, A. S. Bondarenko, U. Grønbjerg, J. Rossmeisl, I. Chorkendorff, Energy Environ. Sci. 2012, 5, 6744.
[2] a) H. A. Gasteiger, P. N. Ross, J. Phys. Chem. 1996, 100, 6715; b) J. Schmidt, V. Stamenkovic, P. N. Ross, Phys. Chem. Chem. Phys. 2003, 5, 400.

[3] US Geological Survery, Mineral Commodities Summaries 2015 http:// minerals.usgs.gov/minerals/pubs/mcs/2015/mcs2015.pdf.

[4] a) B. B. Blizanac, P. N. Ross, N. M. Markovic, J. Phys. Chem. B 2006, 110, 4735 ; b) G. K. H. Wiberg, K. J. J. Mayrhofer, M. Arenz, Fuel Cells 2010, 10, 575.

[5] A. Holewinski, J. Idrobo, S. Linic, Nat. Chem. 2014, 6, 828.

[6] a) A. Holewinski, S. Linic, J. Electrochem. Soc. 2012, 159, H864; b) H. Xin A. Holewinski, S. Linic, ACS Catal. 2012, 2, 12; c) H. Xin, A. Holewinski, N. Schweitzer, E. Nikolla, S. Linic, Top. Catal. 2012, 55, 376

[7] a) Y. Lu, N. Zhang, L. An, X. Li, D. Xia, J. Power Sources 2013, 240, 606; b) Y. Jin, F. Chen, Y. Lei, X. We, ChemCatChem 2015, 7, 2377.

[8] a) C. L. Lee, H. P. Chiou, C. M. Syu, C. C. Wu, Electrochem. Commun. 2010, 12,1609 ; b) J. Ohyama, Y. Okata, N. Watabe, M. Katagiri, A. Nakamura, H. Arikawa, K. I. Shimizu, T. Takeguchi, W. Ueda, A. Satsuma, J. Power Sources 2014, 245, 998; c) Q. Wang, X. CUI, W. Guan, L. Zhang, X. Fan, Z. Shi, W. Zheng, J. Power Sources 2014, 269, 152; d) Y. Lu, Y. Wang, W. Chen, J. Power Sources 2011, 196, 3033; e) P. Christopher, S. Linic, J. Am. Chem. Soc. 2008, 130, 11264; f) P. Christopher, S. Linic, ChemCatChem 2010, 2, 78; g) S. Linic, P. Christopher, ChemCatChem 2010, 2, 1061.

[9] a) J. Greeley, J. Rossmeisl, A. Hellman, J. K. Nørskov, Z. Phys. Chem. 2007, 221, 1209; b) P. Singh, D. A. Buttry, J. Phys. Chem. C 2012, 116, 10656.

[10] a) M. Rycenga, C. M. Cobley, J. Zeng, W. Li, C. Moran, Q. Zhang, D. Qin Y. Xia, Chem. Rev. 2011, 111, 3669; b) X. Xia, J. Zeng, K. Oetien, L. Qingge, Y. Xia, J. Am. Chem. Soc. 2012, 134, 1793.

[11] L. S. Taylor, F. W. Langkilde, G. Zografi, J. Pharm. Sci. 2001, 90, 888.

[12] a) M. Liu, W. Chen, Nanoscale 2013, 5, 12558; b) Y. Lu, W. Chen, J. Power Sources 2012, 197, 107.

[13] a) E. Herrero, L. J. Buller, H. D. Abruna, Chem. Rev. 2001, 101, 1897; b) E. Kirowa-Eisner, Y. Bonfil, D. Tzur, E. Gileadi, J. Electroanal. Chem. 2003, 552, 171; c) H. Bort, K. Juttner, W. J. Lorenz, J. Electroanal. Chem. 1978, 90, 413.

[14] V. Viswanathan, H. A. Hansen, J. Rossmeisl, J. K. Nørskov, ACS Catal. 2012, 2, 1654.

[15] A. Marimuthu, P. Christopher, S. Linic, J. Phys. Chem. C 2012, 116, 9824

Received: August 11, 2015

Published online on December 4, 2015 